\title{
ON THE DIMENSION OF MODULES AND ALGEBRAS, I
}

\author{
SAMUEL EILENBERG, ${ }^{*}$ MASATOSHI IKEDA \\ and TADASI NAKAYAMA
}

In [5], Ikeda-Nagao-Nakayama gave a characterization of algebras of cohomological dimension $\leqq n$. In a subsequent paper [4] Eilenberg gave an alternative treatment of the same question. The present paper is devoted to the discussion of a number of questions suggested by the results of [4] and [5]. Among others it is shown that the conditions employed in stating the main. results in [4] and [5] are equivalent, so that the main results of these two papers are in accord. Further, the cohomological dimension of a residue-algebra. is studied in terms of that of the original algebra and the (module-) dimension of the associated ideal. The terminology and notation employed here are that of [3].

\section{$\S 1$. Modules and quasi-modules}

Throughout this paper, $A$ will denote an algebra over a commutative ring $K$. It is always assumed that $A$ has a unit, and this unit acts as the identity on all $A$-modules.

In addition to $A$-modules we shall also consider quasi-modules in which it is no longer assumed that the unit element 1 of $A$ operates as the identity; however the unic element $\varepsilon$ of $K$ still operates as the identity. Explicitly a (left) $A$-quasi-module is a $K$-module $A$ together with a homomorphism

$$
\Lambda \otimes{ }_{K} A \rightarrow A
$$

satisfying

$$
\gamma(\lambda a)=(\gamma \lambda) a \quad(\gamma, \lambda \in \Lambda ; a \in A)
$$

where $\lambda a$ is the image of $\lambda \otimes a$.

Clearly each $A$-module is a $A$-quasi-module. Further each $K$-module $A$ may

Received June 21, 1954.

* This work was done while S. Eilenberg was engaged under contract AF-18 (600)-562. 
also be regarded as a $\lambda$-quasi-module with $\lambda a=0$ for all $\lambda \in A, a \in A$. In a sense, these two classes exhaust the picture. Indeed, for each 1 -quasi-module $A$ we have the direct sum decomposition (due to Peirce)

$$
A=1 A+A^{\bullet}
$$

where $A^{\bullet}$ consists of all elements $a \in A$ with $1 a=0$. Clearly $1 A$ is a $A$-module, while $A^{\bullet}$ is just a $K$-module converted into a $A$-quasi-module as above.

A $A$-module $A$ is projective if for every epimorphism (i.e. onto-homomorphism)

$$
\varphi: B \rightarrow A
$$

of 1 -modules, there exists a 1 -homomorphism $\psi: A \rightarrow B$ such that $\varphi \psi=$ identity. $A$ is said to be injective if for each monomorphism (i.e. (into-)isomorphism)

$$
\varphi: A \rightarrow C
$$

of 1 -modules, there exists a $A$-homomorphism $\psi: C \rightarrow A$ with $\phi \varphi=$ identity.

Replacing in the above definitions all modules by quasi-modules we obtain the notions of a projective quasi-module and of an injective quasi-module.

Proposition 1. A 1 -quasi-module $A$ is projective [injective] if and only if $1 A$ is a projective [injective] $A$-module and $A^{\bullet}$ is a projective [injective] $K$ module.

Proof. Let $\varphi: B \rightarrow A$ be an epimorphism of A-quasi-modules. Then $\varphi$ decomposes into two components

$$
\varphi_{1}: 1 B \rightarrow 1 A, \quad \varphi_{2}: B^{\bullet} \rightarrow A^{\bullet}
$$

$A$ map $\psi: A \rightarrow B$ with $\varphi \psi=$ identity exists if and only if such maps exist for $\varphi_{1}$ and $\varphi_{2}$. This yields the desired conclusion.

This proposition implies that a $A$-module $A$ is projective [injective] if and only if it is projective [injective] as a quasi-module.

\section{$\S 2$. The Hochschild quasi-operators}

It will be convenient to denote by $A^{n}$ the $n$-fold tensor product $A \otimes \ldots \otimes A$ where $\otimes=\otimes_{K}$. We may regard $A^{n}$ as a two-sided $A$-module by setting

$$
\begin{aligned}
& \lambda\left(\lambda_{1} \otimes \ldots \otimes \lambda_{n}\right)=\lambda \lambda_{1} \otimes \ldots \otimes \lambda_{n}, \\
& \left(\lambda_{1} \otimes \ldots \otimes \lambda_{n}\right) \lambda=\lambda_{1} \otimes \ldots \otimes \lambda_{n} \lambda .
\end{aligned}
$$


We consider the complex $S(1)$ with

$$
\begin{gathered}
S_{n}(\Lambda)=1^{n+2} \quad n=0,1, \ldots, \\
d\left(\lambda_{0} \otimes \ldots \otimes \lambda_{n+1}\right)=\sum_{i=0}^{n}(-1)^{i} \lambda_{0} \otimes \ldots \otimes \lambda_{i} \lambda_{i+1} \otimes \ldots \otimes \lambda_{n+1}
\end{gathered}
$$

and with the augmentation

$$
\varepsilon: S_{0}(\Lambda)=\Lambda \otimes \Lambda \rightarrow A
$$

given by $\varepsilon\left(\lambda_{0} \otimes \lambda_{1}\right)=\lambda_{0} \lambda_{1}$. This complex is acyclic as can be easily seen using the homotopy operator $\zeta: S_{n}(A) \rightarrow S_{n+1}(A)$ given by $\zeta x=1 \otimes x, x \in S_{n}(A)$.

If $A$ is assumed to be $K$-projective, then each $A^{n}(n>1)$ is easily seen to be a $A \otimes A^{*}$-projective module, where $A^{*}$ is the inverse ring of $A$. Thus in this case $S(A)$ is $A \otimes A^{*}$-projective resolution of $A$. This is the standard complex of $A$ as defined in [3] (Ch. IX, §2).

Now let $A$ be a left $A$-module which is $K$-projective. We consider the complex (of left 1 -modules)

$$
S(A)=S(A) \otimes_{\triangle} A .
$$

It is easy to see that $S(A)$ is a projective resolution of $A$. We have

$$
\begin{aligned}
\mathrm{S}_{n}(A) & =S_{n}(\Lambda) \otimes{ }_{\Lambda} A=\Lambda^{n+2} \otimes{ }_{\triangle} A \\
& =A^{n+1} \otimes A \otimes{ }_{\Lambda} A=A^{n+1} \otimes A .
\end{aligned}
$$

In this notation we have

$$
\begin{aligned}
d\left(\lambda_{0} \otimes \ldots \otimes \lambda_{n} \otimes a\right)= & \sum_{i=0}^{n-1}(-1)^{i} \lambda_{0} \otimes \ldots \otimes \lambda_{i} \lambda_{i+1} \otimes \ldots \otimes \lambda_{n} \otimes a \\
& +(-1)^{n} \lambda_{0} \otimes \ldots \otimes \lambda_{n-1} \otimes \lambda_{n} a .
\end{aligned}
$$

Since the complex $S(A)$ is acyclic, we have

$$
B_{n}(S(A))=Z_{n}(S(A)) .
$$

Consequently we have the exact sequence

$$
0 \rightarrow B_{n}(S(A)) \rightarrow S_{n}(A) \rightarrow \ldots \rightarrow S_{0}(A) \rightarrow A \rightarrow 0 .
$$

Since $S_{i}(A)$ are $A$-projective, it follows that $B_{n}(S(A))$ is $A$-projective if and only if $1 . \operatorname{dim}_{\Lambda} A \leqq n+1$.

In addition to the already present $A$-operators on $S_{n}(A)=\Lambda^{n+1} \otimes A$ we introduce $A$-quasi-operators as follows 
We calculate

$$
\gamma *(\lambda * x)=\gamma * d(\lambda \otimes x)=\gamma d(\lambda \otimes x)=d(\gamma \lambda \otimes x)=(\gamma \lambda) * x
$$

so that indeed we have quasi-operators.

Proposition 2. If $A$ and the left 1 -module $A$ are both $K$-projective then for each $n>0$ the following properties are equivalent:

(i) $1 . \operatorname{dim}_{\mathrm{A}} A \leqq n$,

(ii) the left 1 -module $B_{n-1}(S(A))$ is projective,

(iii) the left $A$-module $1 *\left(A^{n} \otimes A\right)$ is projective,

(iv) the left $A$-quasi-module $A^{n} \otimes A$ is projective.

Proof. The equivalence of (i) and (ii) has already been asserted above. We prove the equivalence of (ii) and (iii) by showing that $B_{n-1}(S(A))$ and $1 *\left(A^{n} \otimes A\right)$ coincide as $A$-modules. We have

$$
\begin{gathered}
1 * x=d(1 \otimes x) \in B_{n-1}(S(A)), \\
d(\lambda \otimes x)=\lambda * x=1 *(\lambda * x) \in 1 *\left(A^{n} \otimes A\right)
\end{gathered}
$$

which shows that $B_{n-1}(S(A))$ and $1 *\left(A^{n} \otimes A\right)$ coincide as groups. Further if $x \in B_{n-1}(S(A))$ then $d x=0$ and thus $(*)$ yields $\lambda * x=\lambda x$ so that the $A$-operators also coincide.

To prove the equivalence of (iii) and (iv) consider the direct sum decomposition

$$
.^{n} \otimes A=1 *\left(A^{n} \otimes A\right)+\left(A^{n} \otimes A\right) \bullet .
$$

Since $A^{n} \otimes A$ is $K$-projective it follows that $\left(A^{n} \otimes A\right)$ is $K$-projective. The conclusion thus follows from Prop. 1.

Remark. If $n=0$ then $B_{-1}(S(A))$ should be interpreted as the image of the augmentation $A \otimes A \rightarrow A$; thus $B_{-1}(S(A))=A$. Further if we interpret $A^{0}=K$ then $A^{0} \otimes A=A$. The quasi-operators are $\lambda * a=d(\lambda \otimes a)=\lambda a$ and coincide with the operators. With these interpretations Prop. 2 remains valid also for $n=0$.

\section{§3. Discussion of $\operatorname{dim} \Lambda$.}

Using the results of $\S 2$ it is now possible to close the gap between [4] and [5]. First we give a glossary translating the terminology used here into 
that of $[5]$ and $[6]$ :

module-module $M$ satisfying $M=1 M$, quasi-module-rnodule,

projective quasi-module- $\left(M_{0}\right)$-module, injective quasi-module-- $\left(M_{u}\right)$-module.

Let $A$ be a $K$-algebra. The (cohomological) dimension of $A$ may be defined as follows: $\operatorname{dim} \Lambda \leqq n$ if and only if the cohomology groups $H^{q}(A, A)$ vanish for all $q>n$ and all two-sided $\Lambda$-modules $A$.

Assume that $K$ is a field and that $(A: K)<\infty$. Let $N$ denote the radical of $A$. The main result of [5] may now be stated as follows:

For $n>0$, the condition

(a)

$\operatorname{dim} \Lambda \leqq n$

is equivalent with the set of two conditions

(b)

$\Lambda / N$ is separable,

(c)

$$
1 *\left(\Lambda^{n-1} \otimes N\right) \text { is projective. }
$$

In view of Prop. 2 (c) is equivalent with

$$
\text { 1. } \operatorname{dim}_{\Lambda} N \leqq n
$$

which is in turn equivalent with

$$
\text { 1. } \operatorname{dim}_{\Lambda}(\Lambda / N) \leqq n \text {. }
$$

This is the form of the result as established in [4]. Actually if $\left(\mathrm{c}^{\prime \prime}\right)$ is used, the main result remains valid also for $n=0$.

Remark. In [5] it is proved also that (a) implies $\left(c_{0}\right) \quad 1 *\left(\Lambda^{n-1} \otimes \mathfrak{l}\right)$ is projective for any left ideal $\mathfrak{l}$ of $\Lambda$.

This is equivalent to

$$
\left(\mathbf{c}_{0}^{\prime}\right) \quad 1 . \operatorname{dim}_{\mathrm{\Lambda}} \uparrow<n
$$

or

$$
\left(\mathrm{c}_{0}^{\prime \prime}\right) \quad 1 \cdot \operatorname{dim}_{\Lambda}(\Lambda / \mathfrak{l}) \leqq n .
$$

This last inequality is a consequence of the general inequality $1 . \operatorname{gl} . \operatorname{dim} A \leqq \operatorname{dim} A$ (see [4], Corollary 5). 


\section{$\S 4$. An inequality}

Let 1 and $\Lambda^{\prime}$ be rings and

$$
\varphi: \Lambda \rightarrow \Lambda^{\prime}
$$

a ring homomorphism. By means of this homomorphism, each left $A^{\prime}$-module may also be regarded as a left $A$-module.

Proposition 3. For each left $A^{\prime}$-module $A$ we have

$$
\text { 1. } \operatorname{dim}_{\Lambda} A \leqq 1 . \operatorname{dim}_{\Lambda^{\prime}} A+1 \cdot \operatorname{dim}_{\mathrm{\Lambda}} A^{\prime} .
$$

Proof. This proposition could be derived directly from a spectral sequence established in [3] (Ch. XVI, §5), however we shall give an elementary inductive proof here.

Let $p=1 \cdot \operatorname{dim}_{\Lambda^{\prime}} A$ and $q=1 \cdot \operatorname{dim}_{\Delta} A^{\prime}$. Clearly we may assume that $p$ and $q$ are finite. For each free $A^{\prime}$-module $F$ we have $1 . \operatorname{dim}_{\Delta} F=q$, and therefore for each direct summand $P$ of $F$ we have $\lim _{\Delta} P \leqq q$. This proves the proposition if $A$ is $A^{\prime}$-projective i.e. if $p=0$.

From here we proceed by induction with respect to $p$. We assume $p>0$ and assume that the proposition holds for $A^{\prime}$-modules $A$ of left dimension (over $\left.A^{\prime}\right)$ smaller than $p$. Let

$$
0 \rightarrow B \rightarrow X \rightarrow A \rightarrow 0
$$

be an exact sequence of $\Lambda^{\prime}$-modules with $X A^{\prime}$-projective. Then

$$
\text { 1. } \operatorname{dim}_{\Lambda^{\prime}} X=0, \quad \text { 1. } \operatorname{dim}_{\Lambda^{\prime}} B=p-1
$$

and therefore by the inductive assumption

$$
\text { 1. } \operatorname{dim}_{\mathrm{\Lambda}} X \leqq p<p+q, \quad \text { 1. } \operatorname{dim}_{\mathrm{\Lambda}} B<p+q .
$$

For each left 1 -module $C$ we have the exact sequence

$$
\operatorname{Ext}_{\Lambda}^{p+q}(B, C) \rightarrow \operatorname{Ext}_{\Lambda}^{p+q+1}(A, C) \rightarrow \operatorname{Ext}_{\Lambda}^{p+q+1}(X, C)
$$

and since the extreme terms are zero, so is $\operatorname{Ext}_{\mathrm{A}}^{p+q+1}(A, C)$. Thus $1 . \operatorname{dim}_{\mathrm{A}} A$ $\leqq p+q$, as required.

Corollary 4. If $A^{\prime}$ is semi-simple, then

$$
\text { 1. } \operatorname{dim}_{\mathrm{\Lambda}} A \leqq 1 . \operatorname{dim}_{\mathrm{\Lambda}} \Lambda^{\prime}
$$

for each left .1'-module $A$. 
Theorem 5. Let $\Lambda$ be a K-algebra over a field $K$ with $(\Lambda: K)<\infty$, and let $\mathfrak{l}$ be a two-sided ideal contained in the radical $N$ of $\Lambda$. Denoting $\Lambda^{\prime}=\Lambda / \mathfrak{l}$, we have

$$
\operatorname{dim} \Lambda \leqq \operatorname{dim} \Lambda^{\prime}+1 . \operatorname{dim}_{\mathrm{A}} \Lambda^{\prime} .
$$

Proof. Let $N^{\prime}=N /$ l. Then $N^{\prime}$ is the radical of $\Lambda^{\prime}$ and $A / N \cong \Lambda^{\prime} / N^{\prime}$. Clearly we may assume that $\operatorname{dim} \Lambda^{\prime}<\infty$. This implies that $\Lambda^{\prime} / N^{\prime}$ is separable (see preceeding section). Since both $A / N$ and $A^{\prime} / N^{\prime}$ are separable it follows from the preceeding section that

$$
\begin{aligned}
\operatorname{dim} A & =1 \cdot \operatorname{dim}_{\Lambda}(\Lambda / N)=1 \cdot \operatorname{dim}_{\Lambda}\left(\Lambda^{\prime} / N^{\prime}\right) \\
\operatorname{dim} \Lambda^{\prime} & =1 \cdot \operatorname{dim}_{\Lambda^{\prime}}\left(\Lambda^{\prime} / N^{\prime}\right) .
\end{aligned}
$$

Thus the desired inequality follows from Prop. 3 with $A=\Lambda^{\prime} / N^{\prime}$.

Remark. If instead of $\mathfrak{I} \subset N$ we have $N \subset \mathfrak{l}$ then Cor. 4 is applicable.

\section{§. Cartan Matrix}

In proving that if $\operatorname{dim} A<\infty$ then $A / N$ is separable an important role is played by the Cartan matrix $M(A)$. In fact, denoting by $A_{L}$ the algebra obtained from $A$ by passing to the algebraic closure $L$ of $K$, it was proved in [4] and [5] that if $\operatorname{dim} A<\infty$ then $\operatorname{det} M\left(\Lambda_{L}\right)= \pm 1$. An algebra $A$ is called primary if $\Lambda / N$ is simple. A direct product (sum) of a finite number of primary algebras is called primarily decomposable. An algebra $A$ is called absolutely primarily decomposable if for each extension $K^{\prime}$ of $K$, the algebra $A_{K^{\prime}}$ is primarily indecomposable. It suffices that this be the case for the algebraic ciosure $L$ of $K$. For a structural characterization of absolutely primarily decomposable algebras see $[1], \S 1$.

Proposition 6. If the algebra $A$ is absolutely primarily decomposable then $\operatorname{dim} \Lambda=0, \infty$.

Proof. Since $\operatorname{dim} A$ remains unchanged under extensions of the ground field we may assume that $K$ is algebraically closed. If is semi-simple (i.e. separable) then $\operatorname{dim} A=0$. We may thus assume that $A$ is not semi-simple. Let $A_{1}$ be one of the primary components of $A$ with a non-zero radical $N_{1}$. Now all the primitive idempotents in $A_{1}$ are isomorphic and if $e_{1}$ is one of them then $e_{1} N_{1} e_{1}$ $\neq 0$. Thus 


$$
\operatorname{det} M\left(\Lambda_{1}\right)=\left(e_{1} \Lambda_{1} e_{1}: K\right)=\left(e_{1} N_{1} e_{1}: K\right)+\left(e_{1}\left(\Lambda_{1} / N_{1}\right) e_{1}: K\right)>1 .
$$

Since $\operatorname{det} M(\Lambda)$ is the product of $\operatorname{det} M\left(\Lambda_{i}\right)$ where $\Lambda_{i}$ runs through all the primary components of $A$ it follows that $\operatorname{det} M(A)>1$. Therefore by the result quoted above we have $\operatorname{dim} \Lambda=\infty$.

There are other situations in which it can be proved that $\operatorname{dim} A=\infty$ by showing that the matrix $M\left(\Lambda_{L}\right)$ is not inversible. The converse however is not true as will be shown by an example. Indeed, we shall construct an algebra $A$ over any field $K$ such that $\operatorname{dim} \Lambda=\infty$ but $\operatorname{det} M\left(\Lambda_{L}\right)=--1$.

Let $K$ be an arbitrary field. Given $\alpha=\left(\alpha_{1}, \ldots, \alpha_{12}\right), \alpha_{i} \in K$, we consider the matrices

$$
\begin{gathered}
m_{1}(\alpha)=\left|\begin{array}{ccccc}
\alpha_{1} & 0 & 0 & 0 & 0 \\
\alpha_{3} & \alpha_{2} & 0 & 0 & 0 \\
\alpha_{4} & 0 & \alpha_{2} & 0 & 0 \\
\alpha_{5} & 0 & 0 & \alpha_{2} & 0 \\
\alpha_{10} & \alpha_{3} & \alpha_{7} & \alpha_{6} & \alpha_{1}
\end{array}\right|, \quad m_{2}(\alpha)=\left|\begin{array}{ccccccc}
\alpha_{2} & 0 & 0 & 0 & 0 & 0 & 0 \\
\alpha_{11} & \alpha_{2} & 0 & 0 & 0 & 0 & 0 \\
\alpha_{8} & 0 & \alpha_{1} & 0 & 0 & 0 & 0 \\
\alpha_{7} & 0 & 0 & \alpha_{1} & 0 & 0 & 0 \\
\alpha_{6} & 0 & 0 & 0 & \alpha_{1} & 0 & 0 \\
\alpha_{12} & 0 & 0 & 0 & 0 & \alpha_{2} & 0 \\
\alpha_{9} & \alpha_{11} & \alpha_{3} & \alpha_{4} & \alpha_{5} & \alpha_{12} & \alpha_{2}
\end{array}\right| \\
m(\alpha)=\left|\begin{array}{cc}
m_{1}(\alpha) & 0 \\
0 & m_{2}(\alpha)
\end{array}\right| .
\end{gathered}
$$

The matrices $m(\alpha)$ form an algebra $\Lambda$ with $(\Lambda: K)=12$. Basis elements $x_{i} \in \Lambda(i=1, \ldots, 12)$ are obtained by taking $x_{i}=m(\alpha)$ where $\alpha_{j}=\delta_{i j}$.

The elements $x_{1}$ and $x_{2}$ are primitive idempotents with $x_{1}+x_{2}=1$. Further computation shows that

$$
\begin{aligned}
& x_{1} \Lambda x_{1}=x_{1} K+x_{10} K, \\
& x_{1} \Lambda x_{2}=x_{6} K+x_{7} K+x_{8} K, \\
& x_{2} \Lambda x_{1}=x_{3} K+x_{4} K+x_{5} K, \\
& x_{2} \Lambda x_{2}=x_{2} K+x_{9} K+x_{11} K+x_{12} K .
\end{aligned}
$$

This implies that the idempotents $x_{1}$ and $x_{2}$ are not isomorphic and thus form a maximal set of non-isomorphic idempotents in $A$. Thus the Cartan matrix of $A$ is

$$
M(\Lambda)=\left|\begin{array}{ll}
2 & 3 \\
3 & 4
\end{array}\right|
$$

with determinant -1 . The ground field $K$ played no role in the argument and the result remains valid for any extension of $K$.

Next consider the $K$-homomorphism $\varphi: A \rightarrow K$ given by 


$$
\varphi(m(\alpha))=\alpha_{9}+\alpha_{10}
$$

We have

$$
\varphi(m(\alpha) m(\beta))=\alpha_{11} \beta_{11}+\alpha_{12} \beta_{12}+\sum_{i=1}^{10} \alpha_{i} \beta_{10-i}
$$

This shows that

$$
\varphi(m(\alpha) m(\beta))=\psi(m(\beta) m(\alpha))
$$

and that if $\varphi(m(\alpha) m(\beta))=0$ for all $m(\alpha)$ then $m(\beta)=0$. Thus the hyperplane $\varphi=0$ contains no left ideals (except zero) and contains all commutators. Thus 1 is a symmetric algebra and therefore also a Frobenius algebra (see [2]). For such algebras it has been proved in [5] that $\operatorname{dim} A=0, \infty$. However $A$ is not semi-simple since $x_{3}, \ldots, x_{12}$ are nilpotent. Thus $\operatorname{dim} A=\infty$.

Remark. The argument that $\operatorname{dim} A=\infty$ remains valid if $K$ is an arbitrary commutative ring (with a unit element). This follows from the generalized treatment of symmetric and Frobenius algebras that will appear in the next paper in this series.

\section{BIBLIOGRAPHY}

[1] G. Azumaya and T. Nakayama, On absolutely uni-serial algebras, Jap. J. Math. 19 (1948), 263-273.

[2] R. Brauer and C. Nesbitt, On the regular representations of algebras, Proc. Nat. Acad. Sci. U.S.A. 23 (1937), 236-240.

[3] H. Cartan and S. Eilenberg, Homological Algebra, Princeton University Press (1954).

[4] S. Eilenberg, Algebras of cohomologically finite dimension, Comment. Math. Helv. (to appear).

[5] M. Ikeda, H. Nagao and T. Nakayama, Algebras with vanishing $n$-cohomology groups, Nagoya Math. 7 (1954),

[6] H. Nagao and T. Nakayama, On the structure of $\left(\boldsymbol{M}_{0}\right)$ - and $\left(\boldsymbol{M}_{u}\right)$-modules, Math. Zeit. 59 (1953), 164-170.

\section{Columbia University}

Osaka University

Nagoya University 